

\section{A atuação do profissional de Relações Públicas em pequenas e médias empresas}

Sergio José Andreucci Junior

- Mestre em Comunicação na área de concentração Comunicação na Contemporaneidade pela Faculdade Cásper Líbero

- Pós-graduado em Gestão Estratégica de Negócios pela Escola de Administração de Empresas de São Paulo (Eaesp) da Fundação Getúlio Vargas (FGV)

- Pós-graduado em Marketing pela Fundação Escola do Comércio Álvares Penteado (Fecap)

- Especialista em Gestão da Qualidade Total pela Universidade Federal de Minas Gerais (UFMG)/Fundação Cristiano Othoni

- Bacharel em Relações Públicas pela Faculdade Cásper Líbero

- Professor do Curso de Relações Públicas da Faculdade Cásper Líbero

- Professor colaborador do curso de pós-graduação em Comunicação e Mercado da Escola de Comunicações e Artes da Universidade de São Paulo (ECA-USP)

- Diretor de Planejamento da Andreucci Comunicação

- Sócio participante do Clube de Criação de São Paulo

- Consultor de Relações Públicas de Duke Energy, Merck Sharp \& Dohme e Cisne Negro Cia. de Dança

- andreucci@andreucci.com.br 


\section{Resumo}

Este artigo tem o objetivo de analisar o mercado e o papel do profissional de Relações Públicas em empresas de micro, pequeno e médio porte. O mercado brasileiro é grande e apresenta amplas e diversificadas possibilidades de atuação do profissional de Relações Públicas. No que tange ao tamanho e ao porte das empresas no Brasil, atualmente temos cerca de cinco milhões de empresas constituídas, sendo que desse total $98,3 \%$ são de micro, pequeno e de médio porte.

PALAVRAS-CHAVE: RELAÇÕES PÚBLICAS - COMUNICAÇÃO - MERCADO - MICRO, PEQUENA E MÉDIA EMPRESA • EMPREENDEDOR

\section{Abstract}

This article aims at analyzing the market and the role of a Public Relations professional in "micro", small and medium-sized companies. The Brazilian market is large and offers vast and diversified opportunities for PR professionals. As regards the size of Brazilian companies, presently there are approximately 5 million companies established in the country, $98 \%$ of which are "micro" (equivalent to U.S. Subchapter 5), small or medium-sized companies.

KEYWORDS: PUBLIC RELATIONS • PR - COMMUNICATION • MARKET • "MICRO", SMALL AND MEDIUM-SIZED COMPANY • ENTREPRENEUR

\section{Resumen}

Este artículo tiene el objetivo de analizar el mercado y el papel del profesional de Relaciones Públicas en empresas de micro, pequeño y mediano porte. El mercado brasileño es grande y presenta amplias y diversificadas posibilidades de actuación para el profesional de Relaciones Públicas. En lo que se refiere al tamaño y porte de las empresas en Brasil, actualmente tenemos cerca de cinco millones de empresas constituidas. De ese total el 98,3\% son de micro, pequeño y mediano porte.

PALABRAS CLAVE: RELACIONES PÚBLICAS - COMUNICACIÓN - MERCAdO - MiCRO, PEQUEÑa 
$\mathrm{E}$ ste artigo busca promover uma reflexão sobre a participação do profissional de Relações Públicas em empresas de micro, pequeno e médio porte. Tradicionalmente, tem-se vislumbrado apenas a atividade de Relações Públicas em organizações de grande porte, porém o mercado dos últimos 15 anos vem mostrando grandes mudanças nas relações e oportunidades de trabalho. A terceirização de grande parte das operações das empresas e a criação de muitas prestadoras de serviços especializados nos mostra uma realidade na qual o profissional de Relações Públicas precisa ter um perfil dinâmico e empreendedor.

O mercado é instável e desafiador, exigindo do relações-públicas um perfil, além de gestor, empreendedor. As mudanças são constantes e devem ser encaradas como oportunidades, e não como ameaças. A estratégia de Relações Públicas aplicada em pequenas e médias empresas deve estar focada na comunicação dirigida e em políticas de relacionamento junto aos públicos de interesse, de forma ágil e segura, para realização de negócios e, com o tempo, buscando outros públicos de acordo com o crescimento e as metas da organização.

Ter postura inovadora e uma visão crítica do mercado são pontos básicos para o enfrentamento dos desafios de um mundo competitivo e sem fronteiras. Afinal, o relações-públicas, além de gestor da Comunicação, também precisa ser um empreendedor? A atividade profissional de Relações Públicas em grandes empresas segue o mesmo padrão e estratégia em pequenas e médias empresas?

A seguir, abordaremos estes pontos analisando o exercício profissional de Relações Públicas em pequenas e médias empresas nos novos tempos.

\section{Relações Públicas e as pequenas e médias empresas}

As empresas de micro, pequeno e médio porte, largamente presentes na esfera econômica brasileira e mundial, costumam trabalhar frente a uma realidade oposta à das grandes organizações. Elas possuem características específicas que exigem do profissional de Relações Públicas conhecimento de seu universo, adaptação à realidade e criatividade nas ações postuladas. Conforme define Simões (1995, p. 168),

"O conhecimento da realidade do mundo dos negócios, do Brasil e do mundo, diz que predominam as micro, pequenas e médias empresas, totalizando cerca de 90\% de todo 
A ATUAÇÃO DO PROFISSIONAL DE RELAÇÕES PÚBLICAS EM PEQUENAS

E MÉDIAS EMPRESAS • SERGIO JOSÉ ANDREUCCI JUNIOR

universo. Entre estas, as microempresas destacam-se, também, com outra estimativa de $90 \%$ das três".

De acordo com os padrões de mercado, o Sebrae (Serviço Brasileiro de Apoio às Micro e Pequenas Empresas) ${ }^{1}$, define e classifica as empresas, como:

- Microempresa: na indústria, até 19 pessoas ocupadas; no comércio e serviços, até 9 pessoas ocupadas;

- Pequena empresa: na indústria, de 20 a 99 pessoas ocupadas; no comércio e serviços, de 10 a 49 pessoas ocupadas;

- Média empresa: na indústria, de 100 a 499 pessoas ocupadas; no comércio e serviços, de 50 a 99 pessoas ocupadas;

- Grande empresa: na indústria, acima de 500 pessoas ocupadas; no comércio e serviços, acima de 100 pessoas ocupadas.

Cabe destacar, ainda, algumas características importantes no formato e estrutura das empresas. Segundo análise realizada por Vidal (1995, p. 38), nas pequenas empresas, é reduzido o número de pessoas que ocupam postos diretivos, sendo muito comum essa tarefa ser desempenhada por uma única pessoa. Outra característica é a centralização de quase todos os assuntos e decisões no dirigente principal. O volume mensal de operações e transações é pequeno, se comparado às empresas de maior porte. Entre outros, o volume mensal de produção de bens ou serviços, compras, vendas, operações financeiras, a quantidade e variedade de itens em estoque e o número de clientes e fornecedores estão na ordem de centenas ou apenas de alguns milhares. O número de empregados, entre os ligados à produção e os ligados à administração, está, geralmente, na ordem de poucas dezenas. O relacionamento direto e pessoal entre dirigentes e funcionários, dirigentes e clientes e dirigentes e fornecedores, e a forte relação entre a direção e a propriedade, são outros pontos que distinguem as organizações de pequeno porte das grandes empresas. Também o fato de pertencerem a um único indivíduo, a famílias ou mesmo pequenas sociedades comerciais, sem recorrerem ao mercado de capitais ou estarem vinculadas a grandes empresas ou grupos financeiros, não possuindo, necessariamente, administração especializada. As pequenas empresas são criadas e operadas à imagem e semelhança de seu empreendedor, que freqüentemente acumula, em especial nas organizações de pequeno porte, as funções técnicas, administrativas, de gestão financeira e de vendas, resultando, desse modo, em características diferenciadas e pessoais para cada empreendimento.

A partir da apresentação deste cenário, torna-se importante ressaltar que o desenvolvimento do trabalho do profissional de Relações Públicas junto a essas empresas ge-

$1<$ www.sebrae.com.br $>$ 


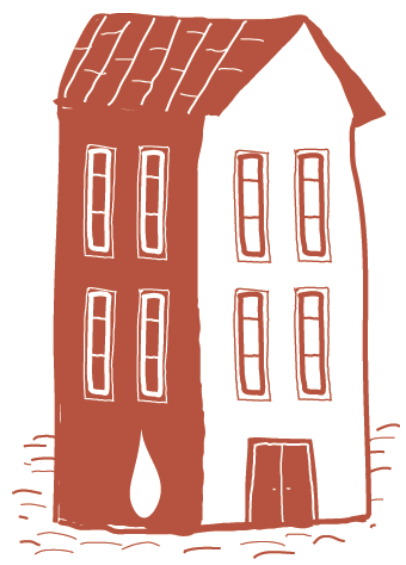

ralmente ocorre por meio da execução de atividades simultâneas e complementares. Além disso, a efetivação do trabalho ocorre quase sempre dentro de orçamentos muito apertados e com poder de decisão restrito. De acordo com Simões (1995, p. 168),

"Uma organização assim gerida não possui estrutura para admitir um profissional encarregado de uma atividade específica de Relações Públicas, e muito menos verbas para financiar programas como os utilizados pelas corporações multinacionais e divulgados através de estudos de casos promocionais, fazendo inveja à maioria dos profissionais da área".

Podemos apontar que as atividades de Relações Públicas frente a essas características precisam ser otimizadas ao máximo, focadas e eficazes. Essa necessidade se dá por questões financeiras, mas também pela pouca exigência de ações estratégicas de longo prazo, uma vez que estas empresas geralmente não possuem estrutura, capital de giro, pessoal, material e espaço físico para um grande aumento na demanda de trabalho, fixando seus esforços somente em ações operacionais de curto prazo.

Embora essas restrições não cheguem a prejudicar o trabalho do profissional de Relações Públicas, ele deve ter o cuidado de direcionar a comunicação de maneira a atingir especificamente seus públicos de interesse, privilegiar a comunicação dirigida e procurar soluções economicamente viáveis, dentro de uma relação custo x benefício equilibrada e de acordo com os objetivos propostos.

O profissional de Relações Públicas, independentemente do tamanho da organização em que trabalha, desenvolve funções específicas e adaptadas à realidade. De acordo com o Conselho Federal de Relações Públicas (Conferp) ${ }^{2}$, em documento intitulado Conclusões do Parlamento Nacional de Relações Públicas, estudo resultante de diversos fóruns realizados por todos os Conselhos Regionais de Relações Públicas (Conrerps) no período de 1992 a 1998 e que teve como objetivo principal a revisão e atualização do

2 Apud KUNSCH, Margarida M. Krohling. Planejamento de Relações Públicas na Comunicação Integrada. São Paulo: Summus Editorial, 2003. p. 126. 
A ATUAÇÃO DO PROFISSIONAL DE RELAÇÕES PÚBLICAS EM PEQUENAS

E MÉDIAS EMPRESAS • SERGIO JOSÉ ANDREUCCI JUNIOR

conteúdo da Lei 5.377 de 11.12.1967, que regulamentou a profissão, foram definidas como funções básicas específicas de Relações Públicas:

- Diagnosticar o relacionamento das entidades com seus públicos;

- Prognosticar a evolução da reação dos públicos diante das ações das entidades;

- Propor políticas e estratégias que atendam às necessidades de relacionamento das entidades com seus públicos;

- Implementar programas e instrumentos que assegurem a interação das entidades com seus públicos.

E de acordo com essas funções específicas, atribuiu como atividades de Relações Públicas:

- Realizar pesquisas e auditorias de opinião e imagem; diagnósticos de pesquisas de auditoria de opinião e imagem; planejamento estratégico de Comunicação Institucional; pesquisa de cenário institucional;

- Estabelecer programas que caracterizem a comunicação estratégica para criação e manutenção do relacionamento das instituições com seus públicos de interesse;

- Planejar, coordenar e executar programas de interesse comunitário; de informação para a opinião pública; de comunicação dirigida; utilização de tecnologia de informação aplicada à opinião pública; esclarecimento de grupos, autoridades e opinião pública sobre os interesses da organização;

- Dedicar-se ao ensino de disciplinas de teoria e técnicas de Relações Públicas;

- Avaliar os resultados dos programas obtidos na administração do processo de relacionamento das entidades com seus públicos.

As atividades de Relações Públicas podem ser devidamente trabalhadas nos ambientes organizacionais de pequeno porte, dentro de seu espaço limitado, porém tornamse menos necessárias ações para determinados públicos, como a imprensa, o governo e outros setores de origem macro para suas realidades. A estrutura orçamentária, produção e pessoal, geralmente, não comportam atividades voltadas para estes públicos, e a empresa acaba direcionando seus esforços para públicos específicos e de maior interesse na relação direta com o seu mercado.

O público interno é outro público que tem suas ações restritas em pequenas empresas. A comunicação ocorre geralmente de forma horizontal e não requer muitos instrumentos específicos, pois não tem tantas dificuldades por fatores como quantidade de pessoal, distâncias entre áreas da empresa, comunicação indireta entre administração e colaboradores etc.. De acordo com Simões (1995, p. 168), "As micro, pequenas e médias empresas necessitam de outra concepção de Relações Públicas, mesmo porque seus públicos não são numerosos e, normalmente, elas não se constituem como fonte de notícias para os meios de comunicação de massa”. 
Contudo, percebemos boas oportunidades, pois existem pequenas ações que podem ser realizadas junto aos públicos de interesse da empresa, como fornecedores, clientes e comunidade. As organizações menores devem focar a sua comunicação em políticas de relacionamento, de forma ágil e segura, a fim de viabilizar seus negócios e, assim, gradativamente, partir para ações específicas com outros públicos, de acordo com a sua necessidade e desenvolvimento.

A realização de ações em apoio ao Marketing caracteriza-se como uma das principais formas de atuação do profissional de Relações Públicas em pequenas e médias empresas. A utilização de instrumentos adaptados e estratégias de Relações Públicas podem perfeitamente atender à realidade das empresas menores, em que, na maior parte das vezes, o grande esforço e a capacidade do investimento estão sempre focados para efetivação dos negócios. Conforme define Yanaze ${ }^{3}$,

"podemos considerar Marketing não apenas como um conjunto de atividades que visam ao mercado, mas, essencialmente, como uma 'filosofia empresarial' que, uma vez implementada em todos os niveis hierárquicos da empresa, grande ou pequena, atua como fator diferencial, tanto de sucesso mercadológico como de máxima rentabilização dos investimentos em atividades direcionadas ao mercado, como: pesquisa, propaganda, promoção de vendas, Relações Públicas e merchandising, entre outras".

Podemos perceber claramente a total relação entre Relações Públicas e Marketing, pois enquanto o Marketing proporciona às empresas o posicionamento de mercado e de definição de objetivos e estratégias mercadológicas, as Relações Públicas planejam e executam ações que visam melhorar e aproximar a empresa dos seus públicos.

\section{Relações-públicas gestor e empreendedor}

O papel das Relações Públicas para o desenvolvimento das organizações no mundo e no Brasil sempre foi de grande relevância para o equilíbrio das relações e atuação das empresas no mercado. A formação dos blocos econômicos e, principalmente, o efeito da globalização das relações de negócios entre as empresas do mundo inteiro, sem dúvida alguma, caracterizaram os grandes e maiores impulsos para o aumento e aceleração da participação do profissional de Relações Públicas no mercado de trabalho.

O profissional que se deseja, hoje, dentro de uma organização, seja ela pequena, média ou grande, deverá ter o perfil empreendedor e estar pronto para enfrentar um mercado em constante transformação e novos desafios. Deverá apresentar uma postura

$3 \mathrm{KUNSCH}$, Margarida M. Krohling (org.). Obtendo Resultados com Relações Públicas. São Paulo: Thomson, 2006. p. 64. 

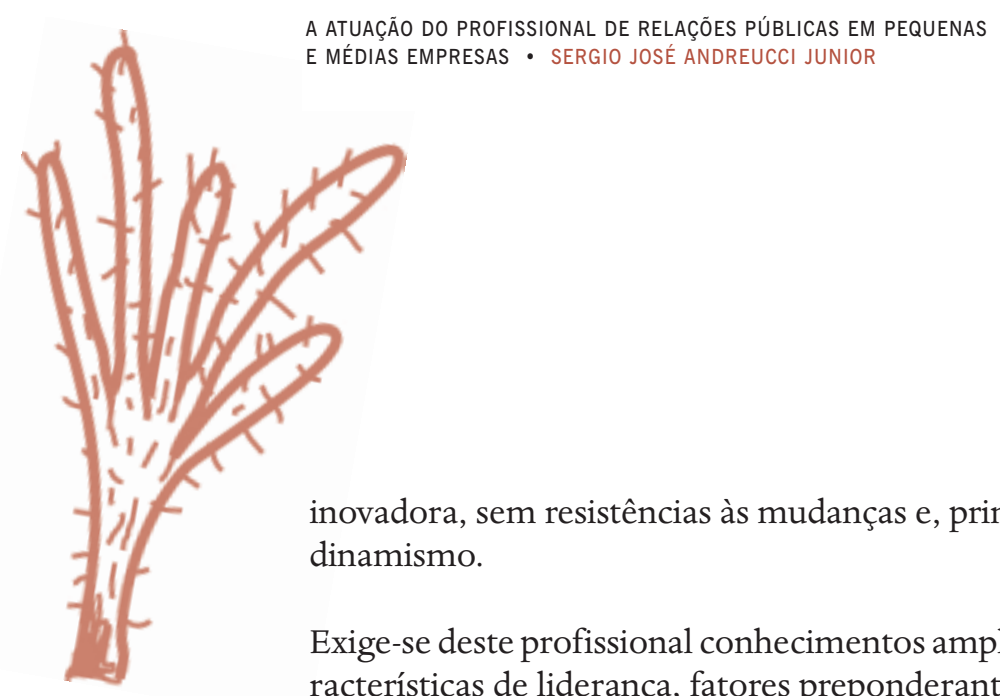

E MÉDIAS EMPRESAS • SERGIO JOSÉ ANDREUCCI JUNIOR

inovadora, sem resistências às mudanças e, principalmente, trabalhar com vontade e dinamismo.

Exige-se deste profissional conhecimentos amplos de mercado, visão abrangente e características de liderança, fatores preponderantes para o desenvolvimento da sua atividade. O profissional de Relações Públicas da atualidade é o gestor dos processos de comunicação da organização, trabalha com as práticas modernas da Administração e, principalmente, com as ferramentas de planejamento. O seu plano de trabalho deverá estar afinado com o planejamento estratégico da organização, ou melhor, o seu plano deverá estar contemplado na formatação final do planejamento estratégico da organização. Não importa o nível hierárquico em que o relações-públicas esteja inserido na organização - seja ele operacional, tático ou estratégico -, deverá contribuir e compor para a efetivação do planejamento estratégico.

O novo profissional de Relações Públicas não é mais aquele que tem emprego e acomodação dentro de uma organização, mas, sim, um profissional que tenha "empregabilidade”, pois as mudanças são rápidas e o mercado exige e procura profissionais dinâmicos. Ter empregabilidade não significa, necessariamente, estar dentro de uma organização e, sim, estar pronto para servir uma organização. As relações de trabalho e as estruturas internas de comunicação das empresas mudaram muito nesses últimos 15 anos. Hoje, encontramos dentro das organizações áreas de comunicação muito enxutas nas quais profissionais são responsáveis pelo planejamento e pela gestão dos processos, ficando grande parte ou quase toda a operacionalização terceirizada, ou seja, a estratégia dentro e a operação fora. Este modelo foi de fato o grande norte da Administração dos últimos tempos: as organizações buscam custos menores e mais efetividade no trabalho e nos resultados.

A participação do profissional de Relações Públicas no mercado ocorre em todos os segmentos da economia, desde empresas ligadas ao setor financeiro, montadoras automotivas, siderúrgicas, indústrias, prestadoras de serviços públicos, órgãos e empresas ligadas ao governo, comércio, universidades, associações e organizações não-governamentais, laboratórios, hospitais, hotéis e empresas ligadas ao turismo, sindicatos, clubes e empresas de entretenimento, mercado cultural, agências de propaganda, assessorias de comunicação e de imprensa, entre outras. O relações-públicas tanto trabalha para uma grande multinacional como para uma pequena empresa de prestação de serviços. 
O fato é que, independentemente do porte da organização e do seu segmento de atuação, percebemos cada vez mais a necessidade da atividade de Relações Públicas. Até pouco tempo atrás, podíamos afirmar que Comunicação era uma estratégia importante para a competitividade das organizações e também das pessoas. Hoje, constatamos que, além disso, é estratégia de sobrevivência num mercado em que a concorrência perdeu os seus limites territoriais, e no qual contratamos serviços e compramos produtos de qualquer lugar do mundo, com qualidade, tecnologia, segurança, prazo e preço.

O mercado brasileiro é grande e apresenta amplas e diversificadas possibilidades de atuação do profissional de Relações Públicas. No que tange a micro, pequenas e médias empresas no Brasil, dados do IBGE (Instituto Brasileiro de Geografia e Estatística) indicam a existência de cinco milhões de empresas no país. Desse total, 98,3\% são de micro empresas ou empresas de pequeno e médio porte. Calcula-se que, juntas, empregam $60 \%$ das pessoas ocupadas no Brasil, ou mais de 40 milhões de pessoas. Ainda segundo dados do IBGE, estão concentradas no setor de comércio, com $37 \%$ de participação, 35\% na prestação de serviços, $14 \%$ na indústria, $8 \%$ na agropecuária e $4 \%$ na construção civil e em outros setores.

A participação de empresas de menor porte na economia do país também é uma conseqüência da globalização, pois para se tornarem mais eficientes, conforme falamos, muitas empresas optaram por terceirizar as atividades de apoio ao seu negócio. Empregados dessas organizações abriram suas empresas para prestarem serviços ao antigo empregador. De acordo com o Sebrae, enquanto a criação de um novo emprego especializado numa indústria tradicional custa cerca de 10 mil dólares, incluindo investimento em treinamento e tecnologia, no pequeno negócio este valor cai para 3,8 mil dólares. Se a modernização tecnológica e a globalização provocaram, por um lado, a diminuição de vagas de trabalho dentro da empresa, por outro, fizeram surgir uma grande geração de novos empreendedores.

Diante desse cenário, bem como pela formação oferecida hoje pelas escolas e faculdades de Comunicação, percebemos que uma boa parte dos profissionais de Relações Públicas já traz consigo características do empreendedorismo. Estar à frente de um negócio não o torna, necessariamente, um empreendedor. Segundo Sergio Bulgacov (1999, p. 50), empreendedor é uma pessoa que cria novo negócio em face a risco e incerteza, com o propósito de conseguir lucro e crescimento, mediante identificação de oportunidades de mercado e agrupamento dos recursos necessários para capitalizar sobre estas oportunidades. Aqueles que são, de fato, empreendedores, têm capacidade de enxergar na adversidade uma oportunidade valiosa. Na visão de Gerber (1990, p. 22), "o empreendedor é o visionário dentre nós. O catalisador de mudanças". Trata-se de uma personalidade criativa, "sempre lidando melhor com o desconhecido, perscrutando o futuro, transformando possibilidades em probabilidades, caos em harmonia". 
A ATUAÇÃO DO PROFISSIONAL DE RELAÇÕES PÚBLICAS EM PEQUENAS

E MÉDIAS EMPRESAS • SERGIO JOSÉ ANDREUCCI JUNIOR

O empreendedor é visto como uma pessoa com desejo por responsabilidade. Não é um jogador nato, trabalha riscos calculados. É, de maneira geral, otimista em relação ao seu sucesso e trabalha muito para alcançá-lo. Ele busca oportunidades e seu olhar está sempre à frente, pouco preocupado com o dia anterior.

Não importa se o profissional de Relações Públicas está dentro ou fora de pequenas e médias organizações, pois o mais relevante é que a nossa atividade profissional, sendo empregado ou prestador de serviços, é de extrema importância. Seja em apoio ao Marketing dentro da empresa, ou como prestador de serviços de consultoria ou assessoria em projetos específicos fora da empresa, percebemos oportunidades e um campo de trabalho bastante amplo.

A receita é simples, porém requer esforço contínuo, disciplina e muita determinação. Espera-se de um relações-públicas atuante em pequenas e médias empresas, além de uma visão abrangente da empresa e do mercado onde atua, uma postura proativa em suas ações. A diversidade entre assuntos financeiros, de Marketing e de negócios passa a conviver todos os dias com o profissional de Relações Públicas, pois além de responder pela gestão da Comunicação Integrada em ações geralmente adaptadas para o porte da empresa, ele fica muito próximo das grandes decisões e, conseqüentemente, torna-se co-responsável pelos resultados finais.

Buscar a simplicidade das coisas mais óbvias em primeiro lugar, ou seja, fazer bem feito o "feijão com arroz" de todos os dias é primordial. A inovação e o empreendedorismo não são resultantes da sorte e, sim, do conhecimento, da lição de casa bem feita e da ação realizada na hora certa.

\section{Bibliografia}

BULGACOV, Sergio (org.) Manual da gestão empresarial. São Paulo: Atlas, 1999.

CAHEN, Roger. Comunicação empresarial. São Paulo: Rocco, 1995.

CESCA, Wilson; CESCA, Cleusa G. Gimenes. Estratégias empresariais diante do novo consumidor. São Paulo: Summus, 2000.

CHIAVENATO, Idalberto. Comportamento organizacional: a dinâmica e o sucesso das organizações. São Paulo: Thomson, 2004.

GERBER, Michael E. O mito do empreendedor. São Paulo: Saraiva, 1990.

GOMES, Nelson; NASSAR, Paulo. A comunicação da pequena empresa. 4. ed. São Paulo: Globo, 1999.

KUNSCH, Margarida M. Krohling (org.). Obtendo resultados com relações públicas. São Paulo: Thomson, 2006.

Planejamento de relações públicas na comunicação integrada. São Paulo: Summus, 2003. 
A ATUACÃO DO PROFISSIONAL DE RELAÇÕES PÚBLICAS EM PEQUENAS

E MÉDIAS EMPRESAS • SERGIO JOSÉ ANDREUCCI JUNIOR

KUTTNER, Robert. Tudo à venda: as virtudes e os limites do mercado. São Paulo: Companhia das Letras, 1998.

LOBOS, Julio. Encantando o cliente: externo e interno. São Paulo: J. Lobos, 1993.

SIMÕES, Roberto Porto. Relações públicas: função política. São Paulo: Summus, 1995.

VIDAL, Antonio Geraldo da Rocha. Informática na pequena e média empresa. São Paulo: Pioneira, 1995.

WEY, Hebe. O processo de relações públicas. São Paulo: Summus, 1986.

Internet

$<$ http://www.fiesp.com.br> $<$ http://www.ibge.gov.br>

$<$ http://www. sebrae.com.br>

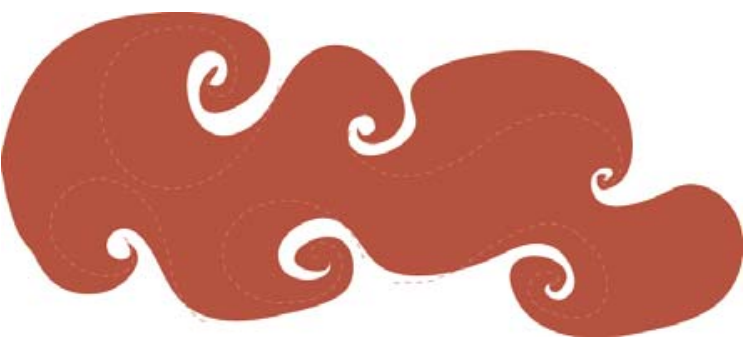

ANO $3 \cdot$ NÚMERO $5 \cdot 2^{\circ}$ SEMESTRE DE $2006 \cdot$ organicom • 131 